\title{
Improved survival of patients with hepatocellular carcinoma and compensated hepatitis $C$ virus-related cirrhosis who attained sustained virological response
}

\author{
Savino Bruno ${ }^{1}$ (D) | Vito Di Marco ${ }^{2}$ | Massimo lavarone ${ }^{3}$ (D) | Luigi Roffi ${ }^{4}$ | \\ Vincenzo Boccaccio ${ }^{1}$ | Andrea Crosignani ${ }^{5}$ | Giuseppe Cabibbo ${ }^{2}$ | Sonia Rossi ${ }^{1}$ | \\ Vincenza Calvaruso $^{2}$ (D) | Alessio Aghemo ${ }^{3}$ | Luca Giacomelli ${ }^{6}$ | Antonio Craxì ${ }^{2}$ | \\ Massimo Colombo $^{3}$ | Patrick Maisonneuve ${ }^{7}$
}

${ }^{1}$ Humanitas University and IRCCS Istituto Clinico Humanitas, Rozzano, Italy

${ }^{2}$ Gastroenterology and Hepatology Unit, Di.Bi.M.I.S., University of Palermo, Palermo, Italy

${ }^{3}$ Gastroenterology and Hepatology Unit, Fondazione IRCCS Cà Granda Ospedale Maggiore Policlinico, Milan, Italy

${ }^{4}$ Department of Medicine, ASST Nord Milano, Milan, Italy

${ }^{5}$ Department of Internal Medicine, A.O. Santi Paolo e Carlo, Milan, Italy

${ }^{6}$ Department of Surgery and Integrated Diagnostics, University of Genoa, Genoa, Italy

${ }^{7}$ Division of Epidemiology and Biostatistics, European Institute of Oncology, Milan, Italy

\section{Correspondence}

Savino Bruno, MD, Humanitas University and IRCCS Istituto Clinico Humanitas, Rozzano, Italy.

Email: savino.bruno@hunimed.eu

Handling Editor: Vincent Wong

\begin{abstract}
Background: Few studies examined the outcome of patients with hepatitis $C$ virus (HCV)-related cirrhosis who developed hepatocellular carcinoma (HCC). The relative weight as determinant of death for cancer vs end-stage liver disease (ESLD) and the benefit of HCV eradication remain undefined. This multicentre, retrospective analysis evaluates overall survival (OS), rate of decompensation and tumour recurrence in compensated HCC patients treated with interferon (IFN) according to HCV status since HCC diagnosis.

Methods: Two groups of patients with HCV-related cirrhosis and HCC were followed since HCC diagnosis: (i) compensated cirrhotics with prior sustained virological response (SVR) on IFN-based regimens ( $N=19)$; (ii) compensated cirrhotics without SVR (viraemic) ( $\mathrm{N}=156)$.

Results: Over a median follow-up of 3.0 years since the onset of HCC, OS was longer for HCC patients with SVR than for viraemic patients (log-rank $P=.004)$. The 5 -year OS rate was $65.9 \%$ in patients with SVR vs $31.9 \%$ in viraemic patients. Similar trends were reported for hepatic decompensation (log-rank $P=.01$ ) and tumour recurrence (logrank $P=.01$ ). These findings were confirmed at multivariable and propensity score analysis. At propensity analysis, 0/19 compensated patients with SVR died for ESLD vs $7 / 19$ (37\%) viraemic patients $(P=.004)$. HCC mortality was similar in the two groups. Conclusions: Hepatocellular carcinoma patients with prior SVR and compensated cirrhosis at the time of tumour diagnosis have prolonged OS than viraemic patients. Given the lack of cirrhosis progression, no SVR patient ultimately died for ESLD while this condition appears the main cause of death among viraemic patients.
\end{abstract}

KEYWORDS

hepatitis $C$ virus, hepatocellular carcinoma, interferon, survival, sustained virological response 


\section{1 | INTRODUCTION}

Patients with hepatitis C virus (HCV)-related cirrhosis are priority candidates for antiviral treatment, since they have an expected high rate of progression to liver decompensation, hepatocellular carcinoma (HCC), and eventually to death. ${ }^{1}$

The achievement of sustained virological response (SVR) after antiviral treatment results in improved clinical outcomes, including reduced risk of liver events and HCC development, compared with lack of response. ${ }^{2-5} \mathrm{~A}$ recent prospective study by Bruno et al. ${ }^{6}$, with a median follow-up of 9.6 years, evaluated 1802 patients with HCVrelated cirrhosis. Compensated patients with SVR on an interferon (IFN)-based regimen had a survival rate similar to that of the age- and gender-matched general population. These benefits attributable to SVR strongly suggest that eradicative treatment of HCV should be administered to patients with compensated HCV cirrhosis as early as possible, given also the recent introduction of IFN-free regimens based on direct antiviral agents (DAA) which allow SVR in the wide majority of patients. ${ }^{2,6-8}$ At the same time, that study confirmed that SVR is associated with lower, but not negligible, risk of HCC occurrence, ${ }^{6}$ in line with other studies. ${ }^{9-12}$

To our knowledge, prospective studies with a follow-up allowing proper analysis of the outcome of patients who developed HCC after anti-HCV therapy-also in correlation with SVR or surrounding disease status-are lacking. In addition, an important clinical question still remains unanswered ${ }^{13}$ : do patients who developed $\mathrm{HCC}$ benefit of HCV eradication by reducing, as a cause of death, the competing risk of cirrhosis progression that ultimately leads to end-stage liver disease (ESLD) independently from anti-HCC treatment?

The present retrospective analysis of a prospectively defined cohort assessed, in the group of cirrhotic patients reported by Bruno et al. ${ }^{6}$ who had developed HCC during surveillance follow-up, overall survival (OS), rate of decompensation and HCC recurrence according to previous response to interferon (IFN)-based regimens, since HCC diagnosis.

Because treatment of cirrhosis with DAA has now become the standard of care, such information can assist in assessing priority for the vast allocation of financial and healthcare resources devoted to these patients. ${ }^{6,14}$

\section{2 | PATIENTS AND METHODS}

\section{1 | Patients}

Surveillance data from three independent cohorts of Italian patients with HCV-related cirrhosis who were followed in tertiary liver centres were considered. ${ }^{6}$ In brief, the first cohort (Milan-1) included consecutive patients with either compensated (Child-Pugh class A5-A6 according to ${ }^{15}$ ) or decompensated (Child-Pugh B7-B9) HCV-related cirrhosis treated at three liver centres (San Paolo Hospital [Milan, Italy], Fatebenefratelli Hospital [Milan, Italy] and San Gerardo Hospital [Monza, Italy]) in the Milan area between 1989 and 1992. The second

\section{Key points}

- Studies on the long-term outcome of patients who developed HCC after anti-HCV therapy are lacking.

- HCC patients with SVR and compensated cirrhosis at diagnosis have prolonged OS than viraemic patients.

- ESLD is the main cause of death in viraemic patients; SVR patients do not die from it.

- This analysis represents a reference for further studies using DAA therapy, which are mandatory.

cohort (Milan-2) included consecutive compensated cirrhosis patients (Child-Pugh class A5-A6) enrolled at the Liver Unit Fondazione IRCCS Ca'Granda, Ospedale Maggiore (Milan, Italy) in 1997. The third cohort (Palermo) included all cirrhotic HCV patients (Child-Pugh class A5-A6) enrolled at the Liver Unit of the University of Palermo who started IFN-based antiviral therapy from 2001 to 2009.

Among subjects enrolled in the above-mentioned cohorts, the present study analyses only patients with compensated cirrhosis at study entry, who received IFN-based therapy and had later developed HCC during surveillance follow-up. Figure 1 displays the flow-chart of the study.

The diagnosis of cirrhosis (F4 METAVIR, F5-6 Ishak) was either made by liver biopsy or according to clinical criteria. Anti-HCV antibodies, HCV RNA (including genotype) and liver function were assessed according to the practice of each centre at the time of patients' observation. HCC diagnosis and treatment (either with a curative intent or not), including the assessment of efficacy for recurrence of HCC were performed according to clinical guidelines available at the time of patients' evaluation, such as the Barcelona Conference and those issued by the American Association for the Study of Liver Diseases (AASLD). IFN mono-therapy or IFN (pegylated or not) in combination with ribavirin was administered according to the standard practice of each centre at the time of evaluation. ${ }^{6}$

All patients underwent a follow-up programme based on abdominal US every 6 months. Until 2001, patients with a newly detected liver focal lesion underwent computed-tomography and US-guided fine-needle biopsy, and after 2001 diagnosis of HCC was in accordance with the European Association for the Study of the Liver (EASL) 2001 and the American Association for the Study of Liver Diseases 2005 criteria and subsequent updates. ${ }^{16-19}$

\section{2 | Surveillance, treatment and outcome at the time of hepatocellular carcinoma development}

After HCC diagnosis, in all patients nodules and tumour-related complications were evaluated by a multidisciplinary team with management changing in line with updating of clinical guidelines when available and according to the specific expertise of each single Center (eg, Centers with experienced surgeons would likely prefer surgery over locoregional treatments, and vice-versa, especially in BCLC very 


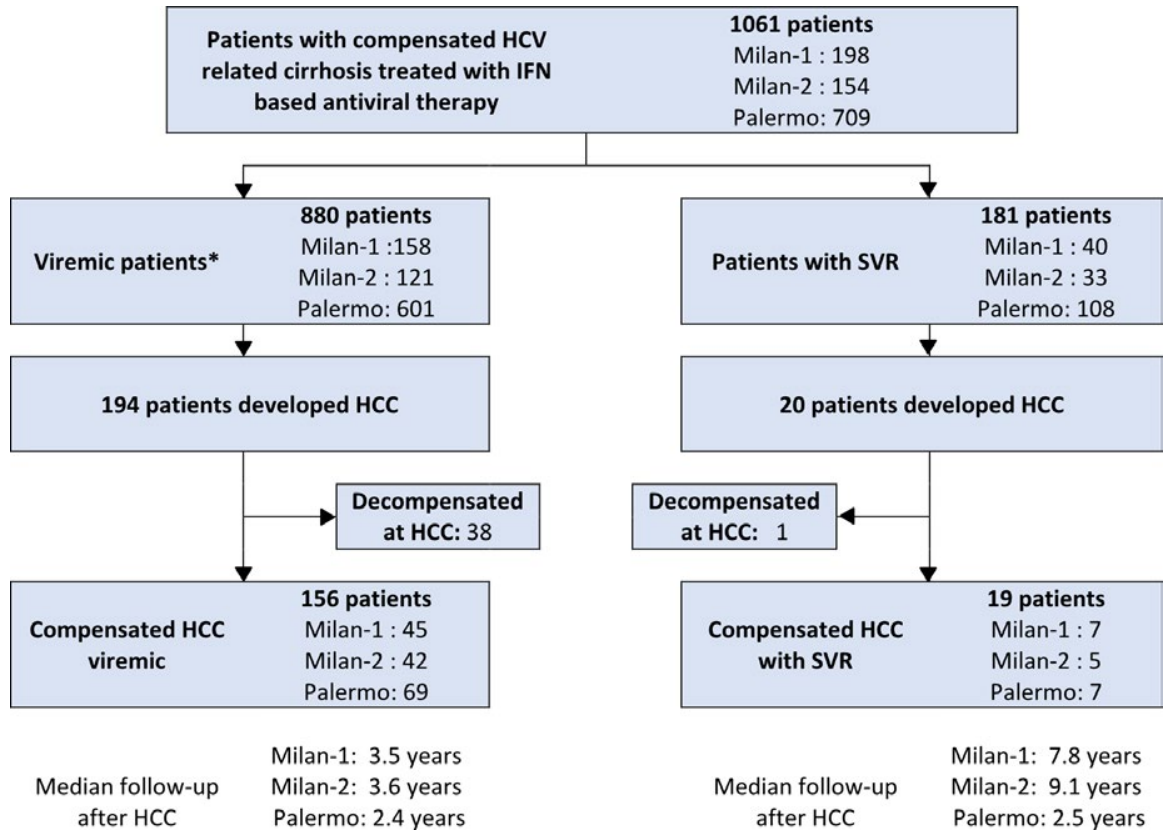

FIGURE 1 Study flowchart early HCC). Moreover, treatment selection took into account also other factors such as tumour site.

After the initiation of HCC treatment, each patient underwent radiological re-evaluation at 1 and 3 months to assess the efficacy of therapy, according to the EASL criteria for radiological response after 2001 and modified Response Evaluation Criteria In Solid Tumors (RECIST) criteria for HCC in more recent years. ${ }^{20}$ Only patients free of disease after the 3-month assessment (considered as complete responders) were considered for late recurrence.

Patients' vital status and cause of death were recorded during surveillance. We defined the causes of death when attributed to ESLD in the absence of HCC as follows: liver failure in Child class CP-C or more, hepato-renal syndrome (HRS), spontaneous bacterial peritonitis with or without sepsis and hepatic encephalopathy. For progression of HCC, we considered BCLC terminal stage D in palliative treatment. For patients lost to follow-up, vital status was updated in 2015 by phone call or in case of non-response from residential town hall vital statistics registry offices.

Data collection and analysis were already approved by all local Institutional Review Committees (Milan 1, Milan 2 and Palermo) at enrolment for the above-mentioned purposes. All patients had signed an informed consent for the use of their personal data for research purposes.

\section{3 | Statistical analysis}

Two groups of patients were considered: (i) IFN-based treated patients with compensated cirrhosis at the time of HCC diagnosis who had prior achieved SVR; (ii) IFN-based treated patients who failed to achieve SVR with compensated cirrhosis at the time of HCC diagnosis (viraemic). Difference in the distribution of patients characteristics between the two groups was assessed by Fisher's Exact test.
The main study outcome was OS, calculated from the date of HCC diagnosis to last contact or death. OS curves were plotted using the Kaplan-Meier method and difference in survival between groups was compared with the log-rank test. Other outcomes were the development of hepatic decompensation and HCC recurrence; these outcomes were also evaluated by Kaplan-Meier analysis. We used Cox proportional hazards regression models to identify characteristics at the time of development of HCC associated with overall mortality, development of hepatic decompensation and HCC recurrence. Multivariable models were constructed, in which factors that did not satisfy the criteria $(P<.10)$ were removed in a step-down phase.

As further confirmatory analysis, patients with SVR at the time of HCC diagnosis were matched using propensity score for demographic and clinical characteristics (age, gender, HCV genotype, albumin blood level, model for end-stage liver disease (MELD) score, gastroesophageal varices, tumour size, Barcelona-Clinic Liver Cancer [BCLC] criteria) with an equal number of viraemic patients. Fisher's exact test was used to assess differences in the distribution of characteristics between the two groups.

All analyses were two-tailed and $P$-values $<.05$ were considered significant. All analyses were performed with the SAS software (version 9.2, Cary, NC, USA).

\section{3 | RESULTS}

\section{1 | Patients}

We identified 175 IFN-based treated patients with cirrhosis who developed an HCC during scheduled surveillance follow-up and were compensated at the time of HCC diagnosis. Of these, 156 had previously failed to achieve SVR after IFN-based therapy, and 19 had prior achieved SVR (Figure 1). 
Patients characteristics at HCC diagnosis in the overall population and stratified according to SVR status are presented in Table 1. The groups differed in terms of HCV genotype $(P=.0006)$, duration of previous IFN treatment $(P=.008)$, serum albumin $(P=.03)$ level, diabetes $(P=.045)$ and type of HCC therapy $(P=.02)$.

\section{2 | Overall survival}

The median observation time was 3 years (range 0.1-24.1). Over this follow-up, 125 patients (71.4\%) patients died; 47 patients $(26.9 \%)$ were still alive at the end of observation while three patients (1.7\%) were lost to follow-up. OS was significantly longer for patients with prior SVR than for viraemic patients (log-rank $P=.004$ ) (Figure 2).

The 5-year OS rate was $65.9 \%(95 \% \mathrm{Cl}$ : $38.9 \%-83.1 \%)$ in patients with SVR and $31.9 \%(24.2 \%-39.8 \%)$ in viraemic patients. Corresponding figures at 10 years were $56.5 \%$ and $16.5 \%$ respectively.

At univariable analysis, viraemic status, older age, class of ChildPugh score, high serum bilirubin, low serum albumin, unmet Milan criteria, increased number and size of liver lesions, BCLC stage and palliative treatment (none or sorafenib-only) were associated with increased mortality, while orthotopic liver transplantation (OLT) was associated with reduced mortality (Table 2). At multivariable analysis, B class of Child (HR=3.17, 95\% Cl: 1.72-5.86), low albumin ( $\leq 3.5 \mathrm{~g} / \mathrm{L})$ ( $\mathrm{HR}=1.67,95 \% \mathrm{Cl}: 1.08-2.57)$, unmet Milan criteria $(\mathrm{HR}=2.71,95 \% \mathrm{Cl}$ : 1.70-4.32), and palliative treatment for $\mathrm{HCC}(\mathrm{HR}=4.25,95 \% \mathrm{Cl}: 1.94-$ 9.29) were independent predictors of mortality, while previous SVR ( $\mathrm{HR}=0.41,95 \% \mathrm{Cl}: 0.19-0.90)$ and $\mathrm{OLT}(\mathrm{HR}=0.13$, 95\% Cl: 0.04-0.50) remained associated with reduced mortality (Table 2 ).

\section{3 | Hepatic decompensation}

Information about hepatic decompensation after the diagnosis of HCC was available for 165/175 patients: 98 developed decompensation. The cumulative incidence of decompensation was significantly higher among viraemic patients than SVR patients (log-rank $P=.01$ ) (Figure 2). The 5-year cumulative rate of decompensation was $35.6 \%(95 \% \mathrm{Cl}$ : $17.5 \%-63.7 \%)$ in patients with SVR and 64.2\% (95\% Cl: 55.5\%-72.7\%) in viraemic patients.

At univariable analysis, absence of SVR, increasing age, low albumin, MELD $\geq 10$, Milan criteria, $\geq 3$ hepatic lesions, intermediate to endstage BCLC and palliative treatment were associated with increased risk of decompensation while OLT was protective (Table 2). At multivariate analysis, SVR ( $\mathrm{HR}=0.35,95 \% \mathrm{Cl}$ : 0.15-0.84), low albumin level ( $\mathrm{HR}=1.81,95 \% \mathrm{Cl}: 1.17-2.80)$, unmet Milan criteria $(\mathrm{HR}=2.40,95 \% \mathrm{Cl}$ : 1.44-4.00) and OLT (HR=0.05, 95\% Cl: 0.01-0.39) were independent predictors of decompensation (Table 2).

\section{4 | Tumour recurrence}

Tumour recurrence was evaluated among 119 compensated patients with $\mathrm{HCC}$ who received treatment with a curative intent, including surgery $(n=20)$, trans-arterial chemoembolization $(n=38)$, percutaneous ethanol injection $(n=13)$ or radio-frequency thermal ablation $(n=48)$ and available information on tumour recurrence.

Time to recurrence was similar for all treatment subtypes (data not shown, $P=.32$ ) but was significantly longer for SVR patients (logrank $P=.01$ ) (Figure 2). At univariable analysis, factors associated with increased recurrence were lack of SVR, HCV genotype 1, presence of gastro-esophageal varices, $\geq 3$ hepatic lesions and intermediate to end-stage BCLC. At multivariable analysis, genotype $1(\mathrm{HR}=2.39$, 95\% Cl: 1.34-4.27), $\geq 3$ hepatic lesions ( $\mathrm{HR}=2.87,95 \% \mathrm{Cl}: 1.29-6.37)$ remained independent predictor of recurrence. After adjustment for these factors, the association with previous achievement of SVR was lost ( $\mathrm{HR}=0.69,95 \% \mathrm{Cl}:$ 0.32-1.50).

\section{5 | Propensity score analyses}

The 19 patients with SVR and compensated cirrhosis at the time of HCC diagnosis were matched with 19 viraemic patients. None of the characteristics evaluated differed between the two groups (Table S1). Patients with SVR had a significantly longer OS than viraemic patients (log-rank $P=.049$ ) (Figure S1). In addition, causes of death differed significantly between the two groups: six patients with SVR ultimately died of HCC compared with four viraemic patients (log-rank $P=.75$ ) while patients with SVR died for ESLD vs 7/19 (37\%) matched viraemic patients $(P=.004)$ (Figure S2).

Patients with SVR had also lower rate of decompensation and tumour recurrence than viraemic patients but the difference was not significant (Figure S1).

\section{4 | DISCUSSION}

This large retrospective analysis of a prospectively defined cohort, conducted in Italian reference liver centres, shows that patients treated with IFN with prior SVR and compensated cirrhosis have prolonged OS after the development of HCC compared with those without SVR. Remarkably, in our study, two of three compensated patients who previously attained SVR were alive 5 years after the development of $\mathrm{HCC}$, while this figure dropped to one out of three in compensated patients who failed to achieve SVR at the time of IFN-based therapy. This advantage is still evident 10 years after HCC diagnosis, as the majority of compensated patients with prior SVR were still alive.

A previous meta-analysis of patients who have undergone surgical resection/ablation of HCC and a retrospective cohort study of a pooled population of patients with and without SVR have suggested similar outcomes. ${ }^{21,22}$ However, to our knowledge the prolonged OS associated with SVR in HCV patients who developed HCC is supported here by the analysis of a prospective cohort for the first time.

Although the low rate of SVR reported in our study-which was expected in the pre-DAA era-should be taken into account, the prolonged OS associated with SVR achievement was confirmed at both univariable and multivariable analysis. In addition, we performed a propensity score analysis to account for the worsened clinical conditions observed in still viraemic patients, as compared with SVR patients. 
TABLE 1 Patients characteristics at the time of development of hepatocellular carcinoma

\begin{tabular}{|c|c|c|c|c|}
\hline & Total & Viraemic & SVR & $\begin{array}{l}P \text {-value fisher } \\
\text { exact test }\end{array}$ \\
\hline All patients & 175 & 156 & 19 & \\
\hline \multicolumn{5}{|l|}{ Center } \\
\hline Milan 1 & 52 & 45 & 7 & \\
\hline Milan 2 & 47 & 42 & 5 & \\
\hline Palermo & 76 & 69 & 7 & .75 \\
\hline \multicolumn{5}{|l|}{ Sex } \\
\hline Male & 126 & 111 & 15 & \\
\hline Female & 49 & 45 & 4 & .59 \\
\hline \multicolumn{5}{|l|}{ Age } \\
\hline$<60$ & 37 & 35 & 2 & \\
\hline $60-69$ & 82 & 72 & 10 & \\
\hline $70+$ & 56 & 49 & 7 & .51 \\
\hline \multicolumn{5}{|c|}{ HCV genotype } \\
\hline 1 & 138 & 130 & 8 & \\
\hline Non-1 & 36 & 26 & 10 & .0006 \\
\hline \multicolumn{5}{|c|}{ IFN duration } \\
\hline$\leq 6 \mathrm{mo}$ & 56 & 51 & 1 & \\
\hline$>6 \mathrm{mo}$ & 115 & 97 & 18 & .008 \\
\hline \multicolumn{5}{|c|}{ Class of Child } \\
\hline$A$ & 144 & 126 & 18 & \\
\hline $\mathrm{B}$ & 18 & 18 & 0 & .23 \\
\hline
\end{tabular}

Serum bilirubin $(\mathrm{mg} / \mathrm{dL})$

$\begin{array}{llcll}\text { Mean } \pm \text { SD } & 1.2 \pm 0.8 & 1.2 \pm 0.9 & 0.9 \pm 0.4 & \\ \begin{array}{l}\text { Low } \\ (<1.2 \mathrm{mg} / \mathrm{dL})\end{array} & 101 & 88 & 13 & \\ \text { High } & 56 & 51 & 5 & .60\end{array}$

$(\geq 1.2 \mathrm{mg} / \mathrm{dL})$

Serum albumin (g/L)

$\begin{array}{lcccc}\text { Mean } \pm \text { SD } & 3.7 \pm 0.6 & 3.7 \pm 0.6 & 4.1 \pm 0.5 & \\ \text { Low }(\leq 3.5 \mathrm{~g} / \mathrm{L}) & 53 & 51 & 2 & \\ \text { High }(>3.5 \mathrm{~g} / \mathrm{L}) & 103 & 87 & 16 & .03\end{array}$

$\begin{array}{lllll}\operatorname{High}(>3.5 \mathrm{~g} / \mathrm{L}) & 103 & 87 & 16 & .03\end{array}$

Prothrombin time (INR)

$\begin{array}{lcccc}\text { Mean } \pm \text { SD } & 1.1 \pm 0.3 & 1.1 \pm 0.3 & 1.1 \pm 0.1 & \\ \text { Low }(\leq 1.2) & 133 & 119 & 14 & \\ \text { High (>1.2) } & 22 & 19 & 3 & .71\end{array}$

$\begin{array}{llll}\text { Mean } \pm \text { SD } & 0.9 \pm 0.2 & 0.9 \pm 0.2 & 0.9 \pm 0.2 \\ \begin{array}{l}\text { Low } \\ (<1.2 \mathrm{mg} / \mathrm{dL})\end{array} & 139 & 123 & 16 \\ \begin{array}{l}\text { High } \\ (\geq 1.2 \mathrm{mg} / \mathrm{dL})\end{array} & 12 & 10 & 2\end{array}$

\section{MELD score}

$\begin{array}{lllll}6-7 & 67 & 59 & 8 & \\ 8-9 & 51 & 45 & 6 & \\ 10+ & 32 & 29 & 3 & 1.00 \\ & & & & \text { (Continues) }\end{array}$

TABLE 1 (Continued)

\begin{tabular}{|c|c|c|c|c|}
\hline & Total & Viraemic & SVR & $\begin{array}{l}P \text {-value fisher } \\
\text { exact test }\end{array}$ \\
\hline \multicolumn{5}{|c|}{ Esophageal varices } \\
\hline Absent & 94 & 82 & 12 & \\
\hline Present & 80 & 73 & 7 & .47 \\
\hline \multicolumn{5}{|l|}{ Diabetes } \\
\hline Absent & 132 & 114 & 18 & \\
\hline Present & 26 & 26 & 0 & .045 \\
\hline \multicolumn{5}{|c|}{ Interval IFN-HCC } \\
\hline$<5 y$ & 67 & 63 & 4 & \\
\hline $5-10 y$ & 68 & 59 & 9 & \\
\hline$\geq 10 y$ & 38 & 32 & 6 & .21 \\
\hline \multicolumn{5}{|l|}{ Milan criteria } \\
\hline Met & 135 & 120 & 15 & \\
\hline Unmet & 39 & 35 & 4 & 1.00 \\
\hline \multicolumn{5}{|c|}{ Number of lesions } \\
\hline 1 & 118 & 103 & 15 & \\
\hline 2 & 34 & 32 & 2 & \\
\hline 3 or more & 22 & 20 & 2 & .61 \\
\hline \multicolumn{5}{|l|}{ Tumour size } \\
\hline$<20 \mathrm{~mm}$ & 41 & 36 & 5 & \\
\hline $20-29 \mathrm{~mm}$ & 72 & 67 & 5 & \\
\hline$\geq 30 \mathrm{~mm}$ & 57 & 48 & 9 & .27 \\
\hline \multicolumn{5}{|l|}{$\mathrm{BCLC}$} \\
\hline Very early & 37 & 32 & 5 & \\
\hline Early & 105 & 93 & 12 & \\
\hline Intermediate & 16 & 16 & 0 & \\
\hline Advanced & 9 & 9 & 0 & \\
\hline End-stage & 1 & 0 & 1 & .10 \\
\hline \multicolumn{5}{|l|}{ HCC therapy } \\
\hline None & 19 & 18 & 1 & \\
\hline Surgery & 22 & 15 & 7 & \\
\hline TACE & 47 & 45 & 2 & \\
\hline PEI & 13 & 10 & 3 & \\
\hline RFTA & 54 & 50 & 4 & \\
\hline OLT & 13 & 12 & 1 & \\
\hline Sorafenib & 6 & 5 & 1 & .02 \\
\hline
\end{tabular}

BCLC, Barcelona-Clinic Liver Cancer criteria; HCC, hepatocellular carcinoma; IFN, Interferon; MELD, model for end-stage liver disease; OLT, orthotopic liver transplantation; PEI, percutaneous ethanol injection; RFTA, radio-frequency thermal ablation; TACE, transarterial chemoembolization. Information missing for few subjects (HCV genotype for one patient, duration of antiviral treatment for eight patients, class of child for 13 patients, bilirubin for 18 patients, albumin for 19 patients, INR for 20 patients, creatinin for 24 patients, MELD score for 25 patients, varices for one patient, diabetes for 17 patients, interval between IFN and HCC for two patients, Milan criteria for one patient, number of lesions for one patient, tumour size for five patients, BCLC for seven patients, HCC therapy for one patient). Bold denotes statistical significance. 
(A)

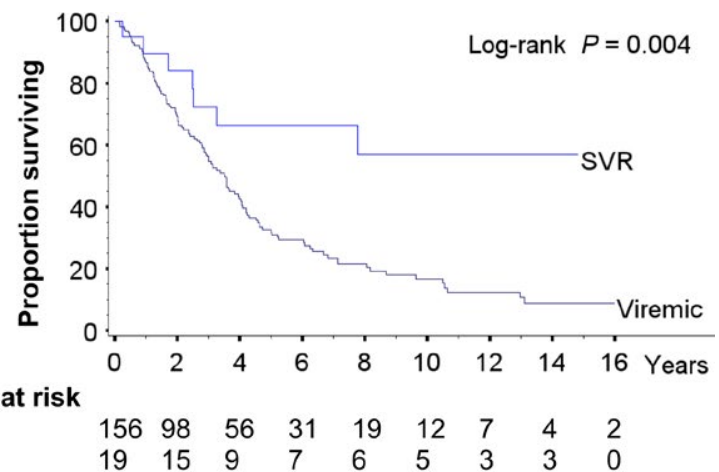

Viraemic

SVR

$\begin{array}{lllllllll}19 & 15 & 9 & 7 & 6 & 5 & 3 & 3 & 0\end{array}$

(B)

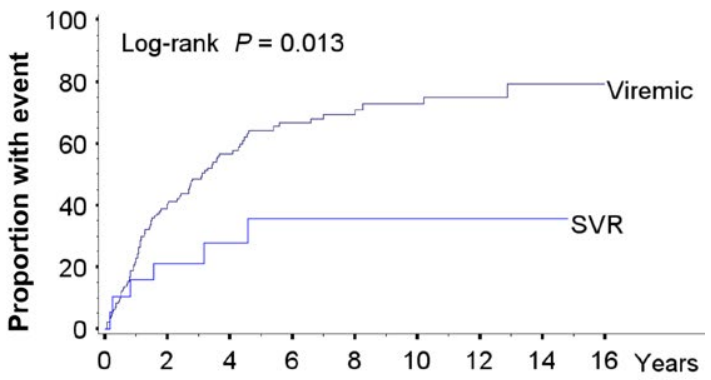

Patients at risk

$\begin{array}{llllllllll}\text { Viraemic } & 146 & 75 & 45 & 27 & 19 & 12 & 6 & 4 & 2 \\ \text { SVR } & 19 & 14 & 10 & 7 & 6 & 5 & 3 & 3 & 0\end{array}$

(C)

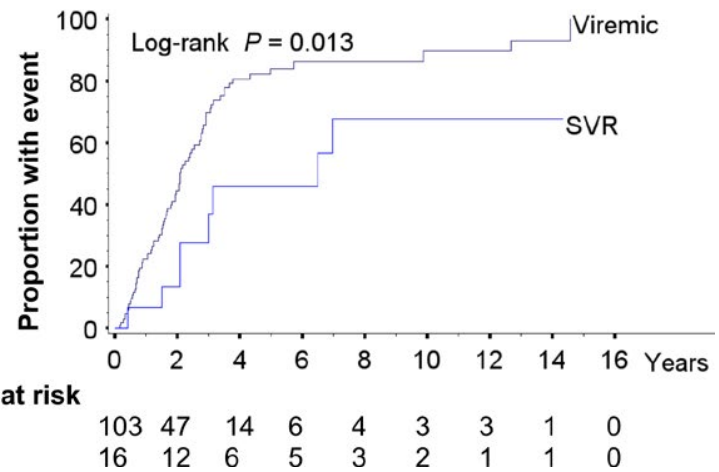

Viraemic

SVR

$\begin{array}{lllllllll}16 & 12 & 6 & 5 & 3 & 2 & 1 & 1 & 0\end{array}$

FIGURE 2 Overall survival (A), time to decompensation (B) and time to $\mathrm{HCC}$ recurrence (C) after HCC among 175 compensated cirrhotic patients according to viraemic status

The results of the propensity analysis confirmed those reported in the main analysis. Remarkably, the propensity analysis showed that the ultimate cause of death significantly differs between viraemic and SVR patients. As a matter of fact, none of patients with SVR died from ESLD, while this latter was the main cause of death in those who failed to achieve SVR. This evidence is in line with a previous analysis which indicated that the onset of HCC in viraemic patients, as well as those of portal hypertension-related complications, quickly accelerates the course of liver disease course to its final stage. ${ }^{23}$

More favourable outcomes were also reported in terms of incidence of hepatic decompensation and time to recurrence, which were improved in the SVR group compared with the viraemic group. However, the benefit on time to recurrence associated with SVR was lost at the multivariable analysis. This could be due to the limited statistical power for this outcome, with only 16 SVR patients included for this analysis, and also to the noted correlation between HCV genotype and SVR. In a multivariate model unadjusted for HCV genotype, the association with SVR remained statistically significant $(H R=0.46 ; 95 \%$ $\mathrm{Cl}$ : 0.22-0.98; $P=.04$ ) after adjustment for other confounders (centre, number of lesions and BCLC).

We must also point out that we had sparse information on tumour recurrence and therefore definite conclusions cannot be made. With respect to recurrence, some recent notes of cautions derived from the results of a small retrospective study which reported a high rate of HCC recurrence after DAA treatment. ${ }^{24}$ However, this study was at least partially flawed by a number of methodological and statistical concerns, and its findings were not confirmed in a large analysis of three prospective studies. ${ }^{25-27}$ Prospective studies on this issue, potentially using new antiviral therapies, may provide more grounded results. Moreover, data on patients with more advanced HCC stages were lacking in our study, since they did not receive IFN-based treatment.

The prolonged OS in eradicated patients is easily justified by the lack of the competing risk of death attributable to concurrent liver disease progression. On the other hand, HCC itself may have a relatively indolent course, while eradication of HCV infection might play a major role in ensuring the best possible clinical outcomes, because multiple anti-tumour procedures can be offered over time to patients with wellpreserved liver function provided by the attainment of SVR. By this way, the best possible management of HCC could be ensured. Given the favourable efficacy/safety ratio of new antiviral therapies and their widespread use in clinical practice, we presume that this advantage will soon much improve, although specific studies on this issue appear warranted. However, interferon might have played a role in reducing $\mathrm{HCC}$ mortality due to its anti-proliferative effect. ${ }^{28}$

\section{1 | Limitations of this study}

This study is not without its limitations. The most important weakness is the small-but reliable-number of patients who achieved SVR, as expected in the pre-DAA era. In addition, the group of SVR patients included subjects who were treated before the occurrence of HCC. Accordingly, patients overall presented more favourable characteristics when compared with those who did not achieve SVR (eg, lower Child-Pugh class and MELD scores, less advanced tumour burden and higher rates of curative treatment) and who were likely to have a shorter duration of infection and less portal hypertension.

We also acknowledge an intrinsic selection bias originated by the use of IFN-based regimens, which have excluded the sickest patients or those with poorest conditions from, treatment.

Last, since IFN-based therapy was successful only in a limited number of patients, with more favourable characteristics, we may not be sure that our findings could fully apply to patients responding to the more recent DAA-based regimens.

Other limitations inherent to any observational retrospective study, such as poor reporting of data (eg, specific data on portal hypertension were not complete and hence this parameter was not considered; data on MELD scores were incomplete), should be also taken into account. 

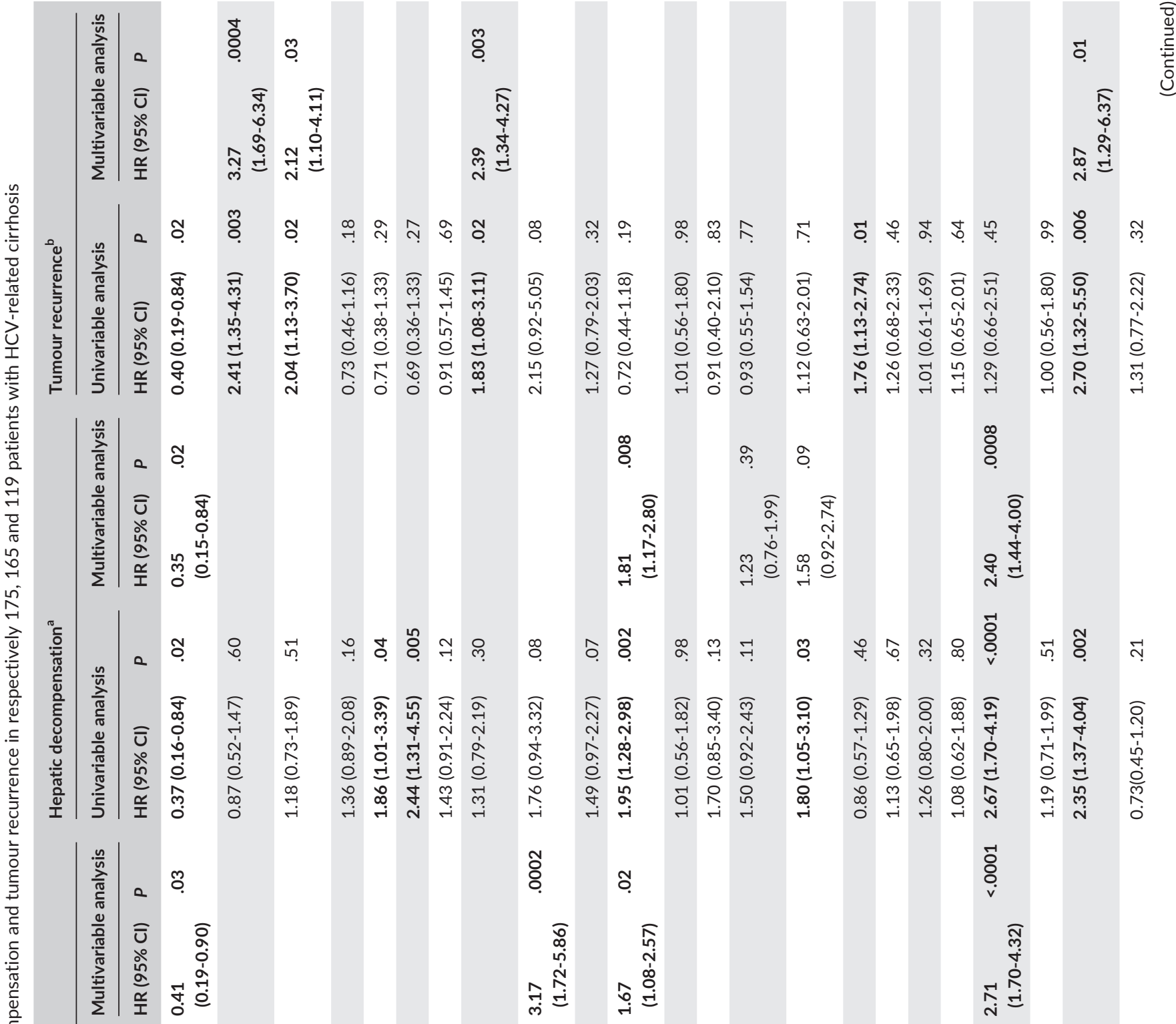

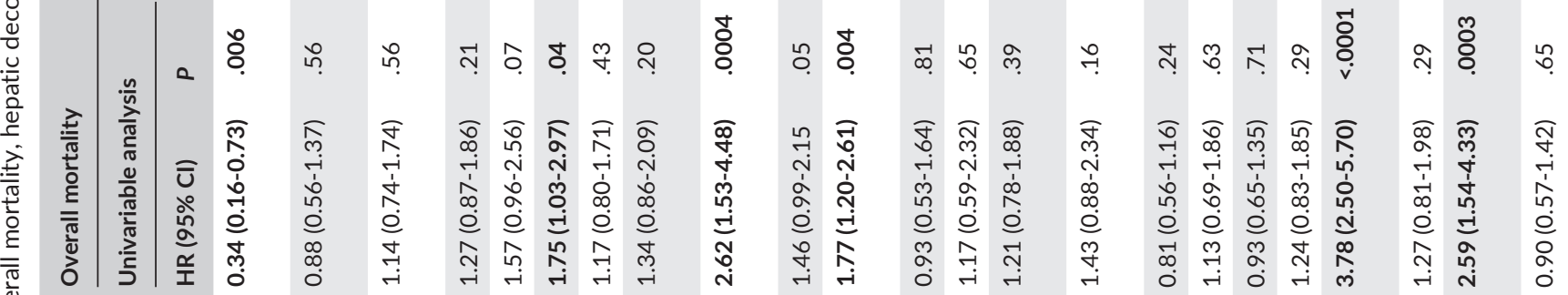

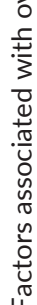

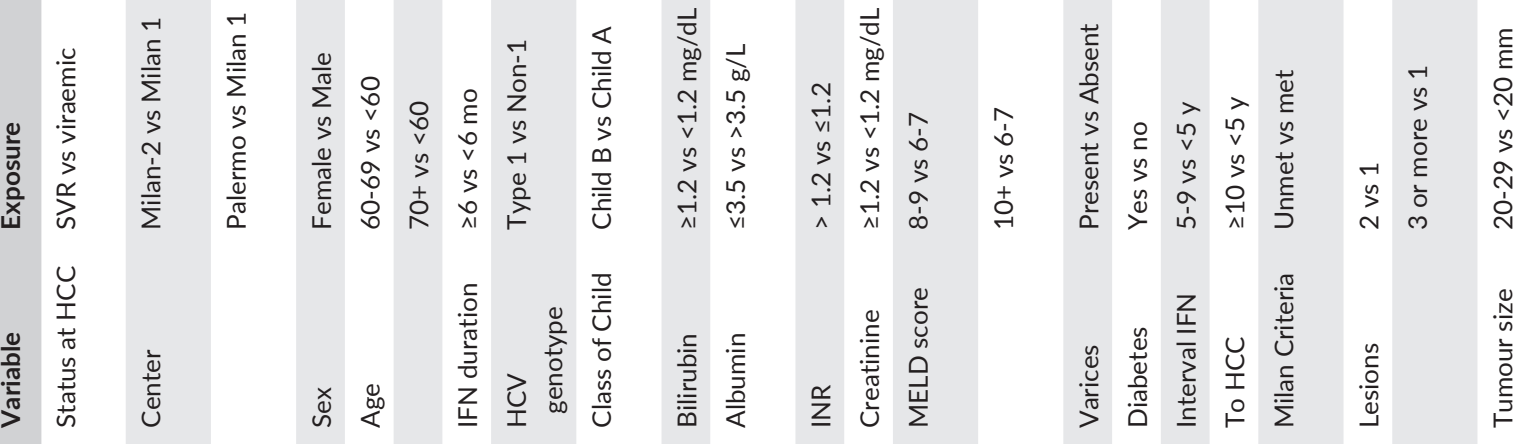




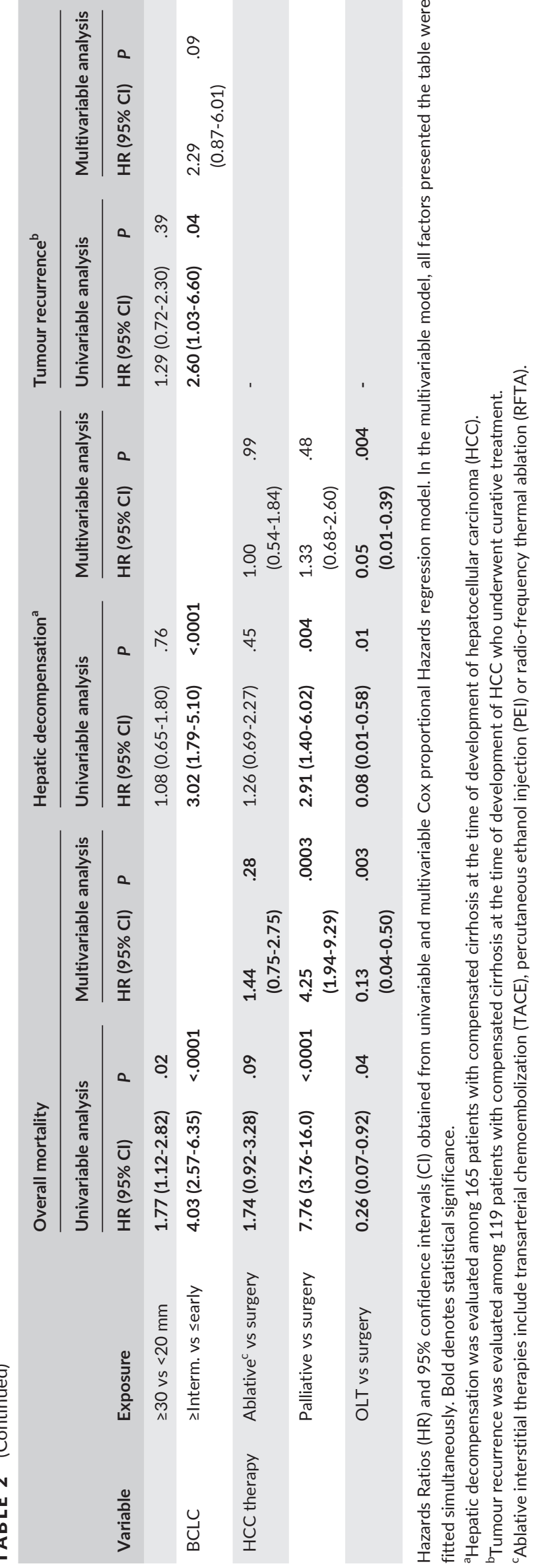

\section{5 | CONCLUSIONS}

Despite the above-mentioned intrinsic and unavoidable limitations, our data show that HCC patients with prior IFN-based achieved SVR and compensated cirrhosis at the time of tumour diagnosis have prolonged OS than viraemic patients. Moreover, ESLD appears to be the main cause of death among viraemic patients, whereas patients with SVR do not die from this condition.

In the next future, due to the widespread use of effective DAA treatments, the ratio of HCC will increase in SVR patients compared with those with virological failure. These patients will be similar to our 19 subjects, and therefore the information provided by our study might represent a reference standard for this population. It is true that the patients enrolled in our cohort were treated with IFN-based regimens. However, given that the biological meaning of SVR is independent from the actual treatment required to attain this goal, ${ }^{12}$ we feel that our data may have a role also in the current DAA era. Indeed, in this illness in which two diseases concur, the virological eradication does cure cirrhosis, thus contributing to improve outcome of HCC patients, especially those with BCLC very early and early HCC and well-compensated cirrhosis. On the other hand, our data cannot be immediately transferred to patients with more advanced HCC or marginally compensated/decompensated cirrhosis.

Because patients in this study were treated years before HCC development and the benefits of HCV therapy are not immediate, new data using dedicated studies on DAA therapy which may support our suggestion appear mandatory. Noteworthy, this study might represent a key reference for further studies in this setting.

\section{ACKNOWLEDGEMENTS}

We thank Ilaria Sogno for useful assistance.

\section{AUTHOR CONTRIBUTION}

Study design: SB, VDM, PM, AC, MC. Experiments and procedures: MI, LR, AC, GC, SR, VC, AA. Data analysis and interpretation: SB, IS, LG, PM. Manuscript drafting: SB, LG. Manuscript editing: All. Approval of final draft: All.

\section{CONFLICT OF INTEREST}

None declared.

\section{REFERENCES}

1. Webster DP, Klenerman P, Dusheiko GM. Hepatitis C. Lancet. 2015;385:1124-1135.

2. Asselah T, Bruno S, Craxi A. HCV cirrhosis at the edge of decompensation: will paritaprevir with ritonavir, ombitasvir, dasabuvir, and ribavirin solve the need for treatment? J Hepatol. 2014;61:1430-1433.

3. Bruno S, Stroffolini T, Colombo M, et al. Italian association of the study of the liver disease (AISF). Sustained virological response to interferon-alpha is associated with improved outcome in HCV-related cirrhosis: a retrospective study. Hepatology. 2007;45:579-587. 
4. Veldt BJ, Heathcote EJ, Wedemeyer H, et al. Sustained virologic response and clinical outcomes in patients with 374 chronic hepatitis $C$ and advanced fibrosis. Ann Intern Med. 2007;147:677-684.

5. EASL. European association for the study of the liver recommendations on treatment of hepatitis C. J Hepatol. 2015;63:199-236.

6. Bruno S, Di Marco V, lavarone M, et al. Survival of patients with HCV cirrhosis and sustained virologic response is similar to the general population. J Hepatol. 2016;64:1217-1223.

7. Boccaccio V, Bruno S. Management of HCV patients with cirrhosis with direct acting antivirals. Liver Int. 2014;34(Suppl 1):38-45.

8. Petta S, Craxì A. Current and future HCV therapy: do we still need other anti-HCV drugs? Liver Int. 2015;35(Suppl 1):4-10.

9. Hsu YC, Wu CY, Lin JT. Hepatitis $C$ virus infection, antiviral therapy, and risk of hepatocellular carcinoma. Semin Oncol. 2015;42:329-338.

10. Shirabe K, Sugimachi K, Harada N, et al. Favorable prognosis in patients with sustained virological response to antiviral therapy, including interferon, for chronic hepatitis $C$ before hepatic resection for hepatocellular carcinoma. Anticancer Res. 2015;35:6963-6969.

11. Kanogawa N, Ogasawara S, Chiba T, et al. Sustained virologic response achieved after curative treatment of hepatitis $C$ virus-related hepatocellular carcinoma as an independent prognostic factor. $J$ Gastroenterol Hepatol. 2015;30:1197-1204.

12. Di Marco V, Calvaruso V, Ferraro D, et al. Effects of viral eradication in patients with hepatitis $C$ virus and cirrhosis differ with stage of portal hypertension. Gastroenterology. 2016;151(130-39):e2.

13. Li DK, Chung RT. Impact of hepatitis C virus eradication on hepatocellular carcinogenesis. Cancer. 2015;121:2874-2882.

14. van der Meer AJ, Wedemeyer $\mathrm{H}$, Feld JJ, et al. Life expectancy in patients with chronic HCV infection and cirrhosis compared with a general population. JAMA. 2014;312:1927-1928.

15. Child CG, Turcotte JG. Surgery and portal hypertension. In: Child CG, ed. The Liver and Portal Hypertension. Philadelphia, PA: Saunders; 1964:50-64.

16. Bruix J, Sherman M, Llovet JM, et al. Clinical management of hepatocellular carcinoma. Conclusions of the Barcelona-2000 EASL conference. European association for the study of the liver. $J$ Hepatol. 2001;35:421-430.

17. Bruix J, Sherman M. Management of hepatocellular carcinoma. Hepatology. 2005;42:1208-1236.

18. Bruix J, Sherman M. Management of hepatocellular carcinoma: an update. Hepatology. 2011;53:1020-1022.

19. European Association For The Study Of The Liver. EASL-EORTC clinical practice guidelines: management of hepatocellular carcinoma. $J$ Hepatol. 2012;56:908-943.
20. Lencioni R, Llovet JM. Modified RECIST (mRECIST) assessment for hepatocellular carcinoma. Semin Liver Dis. 2010;30:52-60.

21. Singal AK, Freeman DH Jr, Anand BS. Meta-analysis: interferon improves outcomes following ablation or resection of hepatocellular carcinoma. Aliment Pharmacol Ther. 2010;32:851-858.

22. Hsu YC, Ho HJ, Wu MS, Lin JT, Wu CY. Postoperative peg-interferon plus ribavirin is associated with reduced recurrence of hepatitis $\mathrm{C}$ virusrelated hepatocellular carcinoma. Hepatology. 2013;58:150-157.

23. Bruno $S$, Zuin $M$, Crosignani $A$, et al. Predicting mortality risk in patients with compensated HCV-induced cirrhosis: a long-term prospective study. Am J Gastroenterol. 2009;104:1147-1158.

24. Reig M, Mariño Z, Perelló C, et al. Unexpected early tumor recurrence in patients with hepatitis $\mathrm{C}$ virus -related hepatocellular carcinoma undergoing interferon-free therapy: a note of caution. J Hepatol. 2016;65:719-726.

25. Pol S. Lack of evidence of an effect of direct acting antivirals on the recurrence of hepatocellular carcinoma: the ANRS collaborative study group on hepatocellular carcinoma (ANRS CO22 HEPATHER, CO12 CIRVIR and CO23 CUPILT cohorts). J Hepatol. 2016;65:734-740.

26. Torres HA, Vauthey JN, Economides MP, Mahale P, Kaseb A. Hepatocellular carcinoma recurrence after treatment with directacting antivirals: first, do no harm by withdrawing treatment. J Hepatol. 2016;65:862-864.

27. Cammà $\mathrm{C}$, Cabibbo G, Craxì A. Direct antiviral agents and risk for HCC early recurrence: much ado about nothing. J Hepatol. 2016;65:861-862.

28. Wang BX, Rahbar R, Fish ENJ. Interferon: current status and future prospects in cancer therapy. J Interferon Cytokine Res. 2011;31:545-552.

\section{SUPPORTING INFORMATION}

Additional Supporting Information may be found online in the supporting information tab for this article.

How to cite this article: Bruno S, Marco VD, lavarone M, et al. Improved survival of patients with hepatocellular carcinoma and compensated hepatitis $C$ virus-related cirrhosis who attained sustained virological response. Liver Int. 2017;37:1526-1534. https://doi.org/10.1111/liv.13452 